\title{
Multiple-Symbol Differential Unitary Space-Time Demodulation with Reduced-Complexity
}

\author{
Tao Cui and Chintha Tellambura \\ Department of Electrical and Computer Engineering \\ University of Alberta \\ Edmonton, AB, Canada T6G 2V4 \\ Email: \{taocui, chintha\}@ece.ualberta.ca
}

\begin{abstract}
We derive a new decision rule for multiple-symbol detection (MSD) of differential unitary space-time modulation (DUSTM). It is valid for both diagonal and non-diagonal constellations, and for quasi-static fading channels. We then present a fast algorithm based on the Extended Euclidean algorithm and bound principles for single symbol detection (SSD) with diagonal constellations. We call it bound-intersection detection (BID) and it is exact maximum likelihood (ML). In high SNR, the complexity of our algorithm is much less than that of the brute-force ML search. We also develop two BID variants for MSD. They are ML but with significantly reduced complexity using branch-and-bound $(\mathrm{BnB})$.
\end{abstract}

\section{INTRODUCTION}

The capacity of wireless communication systems can be substantially enhanced by employing multiple transmit and receive antennas. Space-Time Coding (STC) is designed to realize the benefits of multiple antennas [1]. However, this needs coherent detection using perfect channel state information (CSI), which is difficult to obtain in a fast varying mobile environment and/or in a multiple antenna system. This motivates the research on non-coherent STC detection. Recently, differential space-time modulation (DSTM) has thus received considerable interest [2]-[4]. Hochwald and Sweldens [3] and Hughes [4] have developed a general framework using finite group theory, which is called differential unitary spacetime modulation (DUSTM) in [3]. The finite-group properties simplify the transmitter modulation and constellation design [3]. The diagonal signals, which form an Abelian group and only one transmit antenna is active at any time, are also introduced in [3].

Similar to DPSK in single antenna systems, DSTM suffers a 3-dB performance loss compared with its coherent counterpart. Multiple-symbol detection (MSD) has been proposed for detecting $M$-ary phase-shift keying ( $M$-PSK) signals transmitted over an additive white Gaussian channel [5]. For a moderate number of symbols, MSD bridges the performance gap between $M$-PSK and $M$-DPSK. In [6], MSD and decision-feedback differential detection (DF-DD) [7] are applied to DSTM to overcome the performance loss in fast fading channels and the decision rules are derived. However, the rules can only be applied to diagonal constellations and when the constellation size $L$ is very large, even DF-DD is intractable. For diagonal signals and conventional differential detection, a low-complexity approximate ML detector based on the $L L L$ lattice reduction algorithm [8] has been proposed in [9]. The cosine approximation and the use of $L L L$ make it suboptimal. Moreover, it can not be directly applied for MSD.

In this paper, we derive a new general decision metric for MSD of DUSTM over a quasi-static (QS) fading channel, generalized for both diagonal and non-diagonal differential codes. An ML fast detector for SSD of DUSTM with diagonal signals is derived by using a new bounding approach. Since the decision metric is a sum of non-negative terms, if the sum is less than a bound, the summands generate different candidate sets of the transmit signal. The final candidate set is the intersection of all the sets obtained using the bound. Elements of the candidate set are exhaustively searched until the optimal signal is found. The Extended Euclidean algorithm [10] is used to find the candidate set of integers satisfying the bounds based on the noise variance. We call this algorithm Bound-Intersection Detection (BID) and generalize it for MSD. Further details of this work can be found in our journal paper [11].

\section{System MOdEL}

\section{A. Channel Model}

We consider a MIMO system with $N_{t}$ transmit and $N_{r}$ receive antennas. Each block of the transmitted symbols has $T$ time slots and block interval $T_{B}$. The transmitted symbols during the $n$-th block is denoted by the $T \times N_{t}$ matrix $\mathbf{S}[n]=$ $\left[s_{t, i}[n]\right], t=1,2, \ldots, T$ and $i=1,2, \ldots, N_{t}$, where $s_{t, i}[n]$ is transmitted by the $i$-th antenna in the $t+(n-1) T$-th time slot.

We consider a frequency-flat Rayleigh fading MIMO channel and assume a rich scattering environment. The complex base-band received signal at the $j$-th receive antenna at time slot $t$ in the $n$-th block can be written as

$$
r_{t, j}[n]=\sum_{i=1}^{N_{t}} h_{i, j}[n] s_{t, i}[n]+w_{t, j}[n]
$$

where $h_{i, j}[n]$ denotes the zero mean Gaussian path gain from the $i$-th transmit antenna to the $j$-th receive antenna and $w_{t, j}[n]$ is the complex additive white Gaussian noise at the $j$-th receive antenna. It is assumed that all path gains are 
statistically independent $\left(E\left\{h_{i, j}[n] h_{i^{\prime}, j^{\prime}}^{*}[n]\right\}=0\right)$ and have the same time correlation function $R_{h}(\tau)$. The fading channel is QS, i.e., channel variations within each block are negligible, whereas the channel changes from block to block. Therefore $h_{i, j}[n]$ has correlation $R_{h}[m]=R_{h}\left(m T_{B}\right)$. Typically, when Clarke's model is used, $R_{h}[m]$ is given by

$$
R_{h}[m]=E\left\{h_{i, j}[n] h_{i, j}^{*}[n+m]\right\}=\sigma_{n}^{2} J_{0}\left(2 \pi m f_{d} T_{B}\right)
$$

where $\sigma_{h}^{2}$ denotes the power of the path gain, $J_{0}(\cdot)$ is the zeroth order Bessel function of the first kind, and $f_{d}$ is the Doppler frequency due to users' mobility. Note that the QS condition is met when $f_{d} T_{B}<0.03$. The additive Gaussian noise processes at different receive antennas are also independent and have equal variance $\sigma_{n}^{2}$. Eq. (1) can be written in matrix form as

$$
\mathbf{R}[n]=\mathbf{S}[n] \mathbf{H}[n]+\mathbf{W}[n]
$$

where $\mathbf{R}[n]=\left[r_{t, j}[n]\right]$ is the $T \times N_{r}$ receive matrix, $\mathbf{H}[n]=$ $\left[h_{i, j}[n]\right]$ is the $N_{t} \times N_{r}$ channel matrix, and $\mathbf{W}[n]=\left[w_{t, j}[n]\right]$ is the $T \times N_{r}$ noise matrix.

\section{B. Differential Unitary Space-Time Modulation}

In [3], the signals are modulated by choosing a matrix from a finite group $\mathcal{V}=\left\{\mathbf{V}_{l}, l=0,1, \ldots, L-1\right\}$, where $\mathbf{V}_{l}$ is a $T \times N_{t}$ unitary matrix $\left(\mathbf{V}_{l} \mathbf{V}_{l}^{H}=\mathbf{I}_{T}\right)$ and $L=2^{N_{t} R}$, $R$ denotes the data rate. For DSTM, we assume $T=N_{t}$ and $\mathbf{V}_{0}=\mathbf{I}_{N_{t}}$. The $N_{t} R$ binary information bits are first converted to an integer $l$ within $[0, L-1] . \mathbf{V}[n]=\mathbf{V}_{l}$ is chosen from $\mathcal{V}$. The transmitted symbol at the $n$-th block is encoded as

$$
\mathbf{S}[n]=\mathbf{V}[n] \mathbf{S}[n-1] .
$$

In the first block, $\mathbf{S}[0]=\mathbf{V}_{0}$ is sent. The internal composition property of group ensures that $\mathbf{S}[n] \in \mathcal{V}$ and unitary for any positive $n$. Specifically for diagonal constellations, the unitary matrices $\mathbf{V}_{l}$ are chosen as

$$
\mathbf{V}_{l}=\operatorname{diag}\left\{e^{\jmath 2 \pi u_{1} l / L}, e^{\jmath 2 \pi u_{2} l / L}, \ldots, e^{\jmath 2 \pi u_{N_{t}} l / L}\right\}
$$

where $u_{i}$ for $i=1,2, \ldots, N_{t}$ are optimized to achieve the maximum diversity product [3].

\section{MultiPle-SyMbOL DifFERENTIAL SPACE-Time DEMODULATION}

This section derives the ML MSD metric for DUSTM. The MSD detects the transmitted symbols in $N$ consecutive blocks given $N+1$ received symbols. We consider the sequence from $n=k$ to $n=N+k$. Let $\overline{\mathbf{R}}[k]=\left[\mathbf{R}^{H}[k], \mathbf{R}^{H}[k+\right.$ $\left.1], \ldots, \mathbf{R}^{H}[k+N]\right]$ and $\overline{\mathbf{V}}[k]=\left[\mathbf{V}^{H}[k+1], \mathbf{V}^{H}[k+\right.$ $\left.2], \ldots, \mathbf{V}^{H}[k+N]\right]$. The ML decision rule for the sequence $\overline{\mathbf{V}}[k]$ can be expressed as

$$
\overline{\mathbf{V}}[k]=\underset{\overline{\mathbf{V}}[k]}{\arg \max } f(\overline{\mathbf{R}}[k] \mid \overline{\mathbf{V}}[k])
$$

where $f(a \mid b)$ is the probability density function (pdf) of $a$ conditioned on $b$. The conditional pdf (6) can be calculated by averaging the zero-mean Gaussian pdf

$$
\begin{aligned}
& f(\overline{\mathbf{R}}[k] \mid \overline{\mathbf{V}}[k], \mathbf{S}[k]) \\
= & \frac{1}{\left(\pi^{N N_{t}} \operatorname{det}\left(\mathbf{C}_{R}[k]\right)\right)^{N_{r}}} \exp \left(-\operatorname{tr}\left(\overline{\mathbf{R}}[k] \mathbf{C}_{R}^{-1}[k] \overline{\mathbf{R}}^{H}[k]\right)\right)
\end{aligned}
$$

with respect to the symbol matrix $\mathbf{S}[k]$. The conditional covariance matrix $\mathbf{C}_{R}[k]$ is given by

$$
\begin{aligned}
\mathbf{C}_{R}[k] & =E\left\{\overline{\mathbf{R}}[k] \overline{\mathbf{R}}^{H}[k] \mid \mathbf{S}[k]\right\} \\
& =\overline{\mathbf{S}}_{D}[k] \mathbf{C}_{H} \overline{\mathbf{S}}_{D}^{H}[k]+N_{r} \sigma_{n}^{2} \mathbf{I}_{N_{t}(N+1)}
\end{aligned}
$$

where $\overline{\mathbf{S}}_{D}[k]$ is a block diagonal matrix

$$
\overline{\mathbf{S}}_{D}[k]=\left[\begin{array}{cccc}
\mathbf{S}[k] & & & \\
& \mathbf{S}[k+1] & & \\
& & \ddots & \\
& & & \mathbf{S}[k+N]
\end{array}\right]
$$

and $\mathbf{C}_{H}$ is the covariance matrix of the vector $\overline{\mathbf{H}}=$ $\left[\mathbf{H}^{T}[k], \mathbf{H}^{T}[k+1], \ldots, \mathbf{H}^{T}[k+N]\right]^{T}$ can be represented as

$$
\mathbf{C}_{H}=N_{r}\left(\mathbf{C}_{h} \otimes \mathbf{I}_{N_{t}}\right)
$$

where $\otimes$ denotes the Kronecker product and $\mathbf{C}_{h}$ is given by

$$
\mathbf{C}_{h}=\left[\begin{array}{cccc}
R_{h}[0] & R_{h}[1] & \cdots & R_{h}[N] \\
R_{h}[-1] & R_{h}[0] & \vdots & \vdots \\
\vdots & \vdots & \ddots & \vdots \\
R_{h}[-N] & \ldots & \ldots & R_{h}[0]
\end{array}\right]
$$

Since $\mathbf{S}\left[k^{\prime}\right]$ 's $\left(k^{\prime}=k, k+1, \ldots, k+N\right)$ are unitary matrix, $\overline{\mathbf{S}}_{D}\left[k^{\prime}\right] \overline{\mathbf{S}}_{D}^{H}\left[k^{\prime}\right]=\mathbf{I}_{N_{t}(N+1)}$. Using the distributivity property of Kronecker product, we have

$$
\mathbf{C}_{R}[k]=N_{r} \overline{\mathbf{S}}_{D}[k]\left(\mathbf{C} \otimes \mathbf{I}_{N_{t}}\right) \overline{\mathbf{S}}_{D}^{H}[k]
$$

where $\mathbf{C}=\mathbf{C}_{h}+\sigma_{n}^{2} \mathbf{I}_{N+1}$. It can be readily verified that $\operatorname{det}\left(\mathbf{C}_{R}[k]\right)$ does not depend on the transmitted symbol sequence. Let $\mathbf{A}=\mathbf{C}^{-1}=\left[a_{i, j}\right]$. It can be readily verified that $a_{k_{1}, k_{1}+l}=a_{k_{2}, k_{2}+l}$ for $1 \leq k_{1}, k_{2}<N+1-l$. We normalized $a_{i, j}$ with $-a_{1,2}$ and denote $\tilde{a}_{i, j}=-a_{i, j} / a_{1,2}$. Therefore maximizing (7) is equivalent to minimizing

$$
\begin{aligned}
& \overline{\mathbf{V}}[k]= \\
& \underset{\overline{\mathbf{V}}[k]}{\arg \min } \sum_{i=k}^{k+N-1} \sum_{j=i+1}^{k+N}\left\|\mathbf{R}[j]-\tilde{a}_{i, j}\left(\prod_{m=i+1}^{j} \mathbf{V}[m]\right) \mathbf{R}[i]\right\|_{F}^{2} .
\end{aligned}
$$

Note that (13) does not depend on $\mathbf{S}[k]$. The average over $\mathbf{S}[k]$ in (7) does not need to be computed.

When the channel is static over the $N+1$ blocks, it can be readily obtained $\tilde{a}_{i, j}=1(i=1,2, \ldots, N+1$, $j=1,2, \ldots, N+1$ and $i \neq j$ ). Eq. (13) becomes

$\overline{\mathbf{V}}[k]=\underset{\mathbf{V}[k]}{\arg \min } \sum_{i=k}^{k+N-1} \sum_{j=i+1}^{k+N}\left\|\mathbf{R}[j]-\left(\prod_{m=i+1}^{j} \mathbf{V}[m]\right) \mathbf{R}[i]\right\|_{F}^{2}$. 
When $N=1$, (14) reduces to

$$
\overline{\mathbf{V}}[k]=\underset{\mathbf{\mathbf { V }}[k]}{\arg \min }\|\mathbf{R}[k+1]-\mathbf{V}[k+1] \mathbf{R}[k]\|_{F}^{2} .
$$

When the channel changes in each time slot, the MSDD metric is derived in [6] for diagonal constellations. It can be readily verified that $\mathbf{C}_{R}[k]$ in (12) is the same as (19) in [6]. Therefore, (13) is equivalent to the detection rule (26) in [6] for diagonal constellations though we assume a QS fading channel in the derivation. Hence, the fast detection algorithms in Section IV can also be applied to the noncoherent receivers in [6].

\section{REDUCED COMPLEXITY DIFFERENTIAL UNITARY SPACE-TIME DEMOdULATION}

The fast algorithms in this section are all for diagonal constellations. If the non-diagonal constellation $\tilde{\mathbf{V}}_{l}=\mathbf{U}^{H} \mathbf{V}_{l} \mathbf{U}$ is transmitted [3], where $\mathbf{U}$ is a unitary matrix and $\mathbf{V}_{l}$ is the diagonal constellation, the following reduced-complexity algorithms can be readily modified to this case.

\section{A. Reduced Complexity Single-Symbol Detection}

In [9], the decoding of diagonal differential modulations is converted to the closest vector problem (CVP) in a modular lattice by using the cosine approximation. It is much faster than the ML exhaustive search but the cosine approximation and the $L L L$ algorithm incur performance loss. In the following, we derive a fast and optimal SSD algorithm by using a bounding approach and the Extended Euclidean algorithm.

The ML SSD (15) for diagonal signals can be written as

$$
\hat{l}=\underset{l}{\arg \min }\left\|\mathbf{R}[k+1]-\mathbf{V}_{1}^{l} \mathbf{R}[k]\right\|_{F}^{2}
$$

where

$$
\mathbf{V}_{1}=\operatorname{diag}\left\{e^{\jmath 2 \pi u_{1} / L}, e^{\jmath 2 \pi u_{2} / L}, \ldots, e^{\jmath 2 \pi u_{N_{r}} / L}\right\} .
$$

For simplicity, we drop the time index $k+1$ of $l$. Eq. (16) is then equal to

$$
\begin{aligned}
\hat{l} & =\underset{l}{\arg \min } \sum_{i=1}^{N_{t}} \sum_{j=1}^{N_{r}}\left|r_{i, j}[k+1]-e^{\jmath 2 \pi u_{i} l / L} r_{i, j}[k]\right|^{2} \\
& =\underset{l}{\arg \min } \varphi(l)
\end{aligned}
$$

where $\varphi(l)=\sum_{i=1}^{N_{t}} A_{i}-B_{i} \cos \left[\left(u_{i} l-\phi_{i}\right) 2 \pi / L\right]$,

$$
\begin{aligned}
A_{i} & =\sum_{j=1}^{N_{r}}\left|r_{i, j}[k+1]\right|^{2}+\left|r_{i, j}[k]\right|^{2} \\
B_{i} & =2\left|\sum_{j=1}^{N_{r}} r_{i, j}^{*}[k+1] r_{i, j}[k]\right| \\
\phi_{i} & =\arg \left(\sum_{j=1}^{N_{r}} r_{i, j}[k+1] r_{i, j}^{*}[k]\right) L / 2 \pi .
\end{aligned}
$$

The arg operation takes value in $[0,2 \pi)$ so that $\phi_{i} \in[0, L)$. If $l$ is the true solution, (16) becomes

$$
e=\sum_{i=1}^{N_{t}} \sum_{j=1}^{N_{r}}\left|w_{i, j}[k+1]-e^{\jmath 2 \pi u_{i} l / L} w_{i, j}[k]\right|^{2}
$$

where $w_{i, j}$ is the additive Gaussian noise in (1). Note that $e /\left(2 \sigma_{n}^{2}\right)$ is a chi-square random variable with $2 N_{t} N_{r}$ degrees of freedom. Therefore, we can choose a bound $C$ to be a scaled variance of the noise $C=2 \alpha \sigma_{n}^{2}$ so that the probability that at least one $\hat{l}$ can make (16) less than $C$ is high:

$$
\int_{0}^{\alpha} \frac{x^{N_{t} N_{r}-1} e^{-x / 2}}{\Gamma\left(N_{t} N_{r}\right) 2^{N_{t} N_{r}}} \mathrm{~d} x=1-\epsilon
$$

where $\epsilon$ is set to a value close to 0 (e.g., $\epsilon=0.1$ ). Instead of searching all $0 \leq l<L$, we only search the l's that can make (16) less than $C$. To find all the l's that meet this condition, we note that (18) is the summation of $N_{t}$ non-negative terms. Thus a necessary condition for $l$ to have cost less than $C$ is that each term of (18) is less than $C$, or

$$
A_{i}-B_{i} \cos \left[\left(u_{i} l-\phi_{i}\right) 2 \pi / L\right]<C, i=1,2, \ldots, N_{t} .
$$

Eq. (22) is equivalent to

$\left|\bmod \left(u_{i} l, L\right)-\phi_{i}\right|<\frac{L}{2 \pi} \cos ^{-1}\left(\frac{A_{i}-C}{B_{i}}\right), i=1,2, \ldots, N_{t}$.

The $\bmod (\cdot, L)$ takes value from $[0, L)$. Eq. (23) can be reduced to find all the l's that meet

$$
L B_{i} \leq \bmod \left(u_{i} l, L\right) \leq U B_{i}, i=1,2, \ldots, N_{t}
$$

where

$$
\begin{aligned}
U B_{i} & =\left\lfloor\phi_{i}+\frac{L}{2 \pi} \cos ^{-1}\left(\frac{A_{i}-C}{B_{i}}\right)\right\rfloor \\
L B_{i} & =\left\lceil\phi_{i}-\frac{L}{2 \pi} \cos ^{-1}\left(\frac{A_{i}-C}{B_{i}}\right)\right\rceil .
\end{aligned}
$$

$\lceil\cdot\rceil$ denotes the smallest integer greater than or equal to its argument. $\lfloor\cdot\rfloor$ denotes the largest integer less than or equal to its argument. The problem is to find all the integers within a region in a integer modular ring. The brute-force approach of checking (24) for all $0 \leq l<L$ is not efficient. We thus develop an efficient algorithm to get the values of $l$ satisfying (24).

To develop our solution, we first show how to find $l$ such that $\bmod \left(u_{i} l, L\right)=1$. Since $u_{i}$ is relatively prime to $L$, the gcd of integers $u_{i}$ and $L$ is 1. The well-known Extended Euclidean Algorithm [10] computes gcd of $u_{i}$ and $L$, and additional numbers $d_{i}$ and $k$ such that

$$
u_{i} d_{i}+k L=1
$$

where 1 is the $\operatorname{gcd}$ of $u_{i}$ and $L$. To find $\bmod \left(u_{i} l, L\right)=n$, we multiply both sides of (26) by $n$, which yields

$$
u_{i}\left(n d_{i}\right)+k n L=n .
$$


Therefore $l=\bmod \left(n d_{i}, L\right)$ satisfies $\bmod \left(u_{i} l, L\right)=n$. Let $\mathbf{b}_{i}=\left[L B_{i}, L B_{i}+1, \ldots, U B_{i}\right]$. The candidate set of $l$ for the $i$-th term of (18) is given by

$$
\mathcal{L}_{i}=\bmod \left(d_{i} \cdot \mathbf{b}_{i}, L\right), i=1,2, \ldots, N_{t}
$$

where - denotes element-wise multiplication. Although the Extended Euclidean Algorithm is NP-complete, $d_{i}$ can be computed before detection. For $\mathbf{B}=[0,1, \ldots, L-1]$, we can store $\mathbf{M}=\bmod \left(d_{i} \cdot \mathbf{B}, L\right)$ in memory and (28) can be obtained by $\mathcal{L}_{i}=\left[\mathbf{M}\left(L B_{i}\right), \mathbf{M}\left(L B_{i}+1\right), \ldots, \mathbf{M}\left(U B_{i}\right)\right]$.

The candidates that meet all the $N_{t}$ equations in (22) are chosen, i.e., the candidate set is the intersection of all the $N_{t}$ sets $\mathcal{L}_{i}$ as

$$
\mathcal{L}=\bigcap_{i=1}^{N_{t}} \mathcal{L}_{i}
$$

Intuitively, the term in (22) with the largest $u_{i}$, say $u_{N_{t}}$, varies most with the same change of $l$. Thus, the element in $\mathcal{L}$ that is closest to $\phi_{N_{t}}$ is searched first. If no $l$ can make $\varphi(l)(18)$ less than the bound $C$, we increase the probability $1-\epsilon$ (e.g., $\left.\epsilon=0.1^{2}, 0.1^{3}, \ldots\right)$, adjust the bound $C$ and perform the same process again. If $l^{*}$ is chosen, $C$ is replaced by the new cost $\varphi\left(l^{*}\right)$. The same process is performed again using the new bound $C . l^{*} \in \mathcal{L}$ is selected, Eq. (18) is computed, if it is less than $C, C$ is replaced by the new cost. $l^{*}$ is deleted from the set $\mathcal{L}\left(\mathcal{L}=\mathcal{L}-\left\{l^{*}\right\}\right)$. In the later iterations, (29) is replaced by

$$
\mathcal{L}=\mathcal{L} \cap\left(\bigcap_{i=1}^{N_{t}} \mathcal{L}_{i}\right) .
$$

The process continues until $\mathcal{L}$ becomes the null set. The $l$ with the minimum cost is selected as the optimal solution. We call this optimal detection algorithm Bound-Intersection Detection (BID).

To further improve the performance of BID, we note that each term of (18) has a lower bound as

$$
l b_{i}=A_{i}-B_{i} \cos \left[\Delta \phi_{i} 2 \pi / L\right], i=1,2, \ldots, N_{t}
$$

where $\Delta \phi_{i}=\phi_{i}-\left\lceil\phi_{i}\right\rfloor,\lceil\cdot\rfloor$ denotes the nearest integer to its argument. Hence, the lower and upper bounds (25) are updated to

$$
\begin{aligned}
U B_{i} & =\left\lfloor\phi_{i}+\frac{L}{2 \pi} \cos ^{-1}\left(\frac{A_{i}-C+\sum_{j=1, j \neq i}^{N_{t}} l b_{j}}{B_{i}}\right)\right\rfloor \\
L B_{i} & =\left\lceil\phi_{i}-\frac{L}{2 \pi} \cos ^{-1}\left(\frac{A_{i}-C+\sum_{j=1, j \neq i}^{N_{t}} l b_{j}}{B_{i}}\right)\right] .
\end{aligned}
$$

In high SNR, $C$ is small. The $\mathcal{L}$ in (30) usually contains only one element. On the contrary, $C$ becomes large in low SNR. The size of $\mathcal{L}$ approaches $L$. Therefore the complexity of BID decreases with the increase of SNR.

\section{B. Reduced Complexity Multiple-Symbol Detection}

For the MSD of the diagonal signals, the search space increases to $L^{N}$ and (14) can be reduced to

$$
\begin{aligned}
\hat{\mathbf{l}} & =\left[\hat{l}_{k+1}, \ldots, \hat{l}_{k+N}\right] \\
& =\underset{l_{k+1}, \ldots, l_{k+N}}{\arg \min } \sum_{i=k}^{k+N-1} \sum_{j=i+1}^{k+N}\left\|\mathbf{R}[j]-\mathbf{V}_{1}^{\left({ }^{j}{ }_{m=i+1} l_{m}\right)} \mathbf{R}[i]\right\|_{F}^{2} .
\end{aligned}
$$

where $\mathbf{V}_{1}$ is given in (17). We next give two MSD algorithms which generalize the BID.

1) MSD1: We first use the BID to detect the $N$ block symbols and the result is denoted by $\hat{\mathbf{l}}$, which is then substituted back to (33) and the cost is denoted by $C$. Note that (33) is the summation of non-negative terms. An exhaustive search is performed. After each term in (33) is computed, the current cost is compared with $C$. If it is larger than $C$, the search stops and another candidate is tested. When all the $(N+1) N / 2$ terms have been finished, the total cost is compared with $C$. If the cost is less than $C, C$ is replaced by this value, the current $\mathbf{l}$ is saved and the search continues until all the $L^{N}$ possible candidates have been finished. The best one is output as the optimal solution. This MSD is similar to the Branchand-Bound $(\mathrm{BnB})$ algorithm. The efficiency of this algorithm is low when $L$ is very large and/or in the low SNR region, which makes the initial bound $C$ very loose.

2) MSD2: Our proposed BID is efficient, because it does not need to search all the $0 \leq l<L$. To apply the same idea to MSD, we also begin by using the BID for $N$ blocks SSD. The resulting 1 is then substituted back into (33) and the initial bound is obtained as $C$. Since (33) is the summation of $(N+1) N / 2$ non-negative terms, a necessary condition for (33) to have cost less than $C$ is that each term of (18) is less than $C$, in particular

$$
\left\|\mathbf{R}[k+N]-\mathbf{V}_{1}^{l_{k+N}} \mathbf{R}[k+N-1]\right\|_{F}^{2}<C .
$$

which is the SSD problem. The candidate set $\mathcal{L}_{k+1}$ for $l_{k+N}$ can be found by using BID. For every $l_{k+1}$ in $\mathcal{L}_{k+N}$, the bound for $l_{k+N-1}$ can be improved to $C-B_{k+N}$, where $B_{k+N}=\left\|\mathbf{R}[k+N]-\mathbf{V}_{1}^{l_{k+N}} \mathbf{R}[k+N-1]\right\|_{F}^{2}$. The candidate set $\mathcal{L}_{k+N-1}$ for $l_{k+N-1}$ can also be found by using BID. The similar process continues for $l_{k+N-2}$ and so on. When it comes to $l_{m}$, the bound is updated as $C-\sum_{i=m+1}^{k+N} B_{i}$, where $B_{i}$ is given by

$$
B_{i}=\sum_{j=i+1}^{k+N}\left\|\mathbf{R}[j]-\mathbf{V}_{1}^{\left({ }_{m=i+1}^{j} l_{m}\right)} \mathbf{R}[i]\right\|_{F}^{2} .
$$

When a set of 1 has been chosen, (33) is computed and compared with $C$. If it is less than $C, C$ is updated and the candidate set for each $l_{m}$ is also updated by using the new bound. The process continues until all the elements in the candidate set have been searched. 


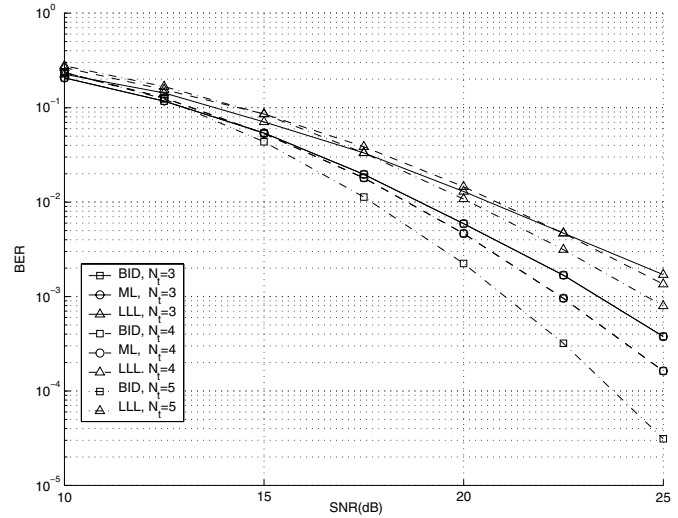

Fig. 1. Performance comparison for $N_{t}=3,4,5$ transmitter antennas, $N_{r}=1$ receiver antenna.

TABLE I

COMPLEXITY COMPARISON FOR ML, LLL AND BID IN FLOPS.

\begin{tabular}{c|c|c|c}
\hline & ML & LLL & BID $(25 \mathrm{~dB})$ \\
\hline$N_{t}=3, N_{r}=1, R=2$ & 2469 & 668 & 214 \\
$N_{t}=4, N_{r}=1, R=2$ & 13312 & 2469 & 572 \\
$N_{t}=5, N_{r}=1, R=2$ & 66560 & 8102 & 2341 \\
\hline
\end{tabular}

To further reduce complexity, the similar idea in BID can be used to find the lower bound of each term in (33), which can be obtained the same as (31). The bound for each $l$ can be further reduced by these lower bounds.

\section{Simulation RESUlts}

We assume that the channel matrix remains approximately constant for $N$ blocks and the fading is independent between antennas. But the channel changes from $N$ blocks to another $N$ blocks. Binary data are transmitted and mapped to the diagonal modulation. The $u_{m}$ 's for the diagonal constellations are selected from Table I in [9].

Fig. 1 compares the performance of BID for SSD with those of ML and lattice decoding algorithm in [9] when $N_{t}=$ $3,4,5, N_{r}=1$ and $R=2$. Our proposed BID is exactly ML, while the gap between ML and the lattice decoder is relatively large. The $L L L$ has a $2-\mathrm{dB}$ gap from $\mathrm{ML}$ at $\mathrm{BER}=0.002$. The Fig. 5 in [9] shows the block error probability and may use the exact algorithm after performing $L L L$, which increases the complexity by $2^{\left(N_{t}+1\right) N_{t} / 4+N_{t}}$. Therefore, we do not use the exact algorithm.

Fig. 2 shows the complexity of the BID in flops when $N_{t}=$ $2,3,4,5, N_{r}=1$ and $R=2$. Since the decoding time depends on the method of programming, the speed of computer etc., we use the flop as the criterion to evaluate the complexity of different algorithms. The flops of different algorithms are obtained by using the MATLAB function "flops". With the increase of SNR, the flops of BID can be reduced (Fig. 2). The complexities of ML and $L L L$ algorithm are almost constant, which are given in Table I for comparison. In high SNR, BID is much faster than both ML and $L L L$ and offers ML performance. Note that the DUST signals are especially effective in high SNR [3], where BID is the most efficient.

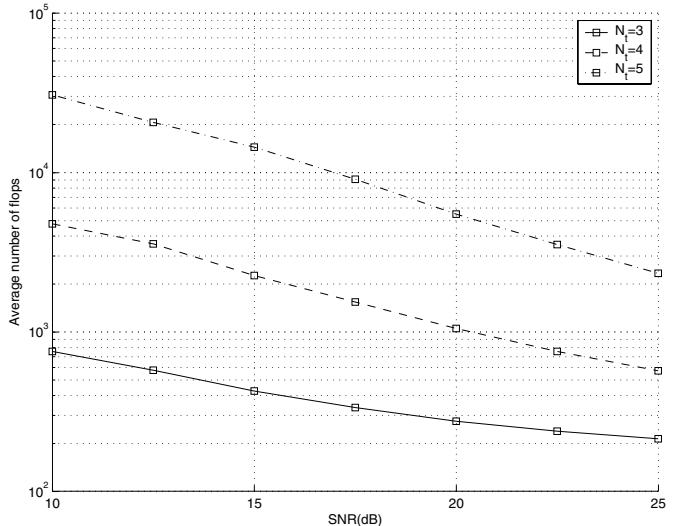

Fig. 2. Complexity of BID for $N_{t}=3,4,5$ transmitter antennas, $N_{r}=1$ receiver antenna.

\section{CONCLUSION}

In this paper, a new decision metric for multiple symbol DUSTM decoding is derived, which is the sum of ML metric for each of the received signals. The new metric can be applied for both diagonal constellations and non-diagonal constellations, and for quasi-static fading channels. A reducedcomplexity detector called BID for single symbol detection with diagonal constellations is developed, which is ML and has low complexity in high SNR. For MSD, two detectors are developed, both of which start from the BID solution. The metric derived in this paper can also be extended to Ricean channels.

\section{REFERENCES}

[1] V. Tarokh, N. Seshadri, and A. R. Calderbank, "Space-time codes for high data rate wireless communication: performance criterion and code construction," IEEE Trans. Inform. Theory, vol. 44, no. 2, pp. 744 765, March 1998.

[2] V. Tarokh and H. Jafarkhani, "A differential detection scheme for transmit diversity," IEEE J. Select. Areas Commun., vol. 18, no. 7, pp. 1169 - 1174, July 2000.

[3] B. M. Hochwald and W. Sweldens, "Differential unitary space-time modulation," IEEE Trans. Commun., vol. 48, no. 12, pp. 2041 - 2052, Dec. 2000.

[4] B. L. Hughes, "Differential space-time modulation," IEEE Trans. Inform. Theory, vol. 46, no. 7, pp. 2567 - 2578, Nov. 2000.

[5] D. Divsalar and M. K. Simon, "Multiple-symbol differential detection of MPSK," IEEE Trans. Commun., vol. 38, no. 3, pp. 300 - 308, March 1990.

[6] R. Schober and L. H. J. Lampe, "Noncoherent receivers for differential space-time modulation," IEEE Trans. Commun., vol. 50, no. 5, pp. 768 - 777, May 2002.

[7] R. Schober, W. H. Gerstacker, and J. B. Huber, "Decision-feedback differential detection of MDPSK for flat Rayleigh fading channels," IEEE Trans. Commun., vol. 47, no. 7, pp. 1025 - 1035, July 1999.

[8] A. Lenstra, H. Lenstra, and L. Lovsz, "Factoring polynomials with rational coefficients," Math. Ann., vol. 261, pp. 515-534, 1982.

[9] K. L. Clarkson, W. Sweldens, and A. Zheng, "Fast multiple-antenna differential decoding," IEEE Trans. Commun., vol. 49, no. 2, pp. 253 261, Feb. 2001.

[10] E. Bach and J. Shallit, Algorithmic Number Theory, Vol. 1: Efficient Algorithms. Cambridge, MA: MIT Press, 1996.

[11] T. Cui and C. Tellambura, "Efficient detection of multiple-symbol differential unitary space-time modulation," submitted to IEEE Trans. on Commun. 